\title{
Norois
}

Environnement, aménagement, société

$232 \mid 2014$

Modes de vie, modes d'habiter des aînés entre inclusion et exclusion

\section{Désacraliser la solidarité familiale. Quand le cancer touche des immigrés âgés}

Desacralisation of family solidarity. When cancer affects elderly immigrants

\section{Anaïk Pian}

\section{OpenEdition}

\section{Journals}

Édition électronique

URL : https://journals.openedition.org/norois/5161

DOI : 10.4000/norois.5161

ISSN : $1760-8546$

Éditeur

Presses universitaires de Rennes

Édition imprimée

Date de publication : 30 octobre 2014

Pagination : 67-80

ISBN : 978-2-7535-3974-7

ISSN : 0029-182X

Référence électronique

Anaïk Pian, «Désacraliser la solidarité familiale. Quand le cancer touche des immigrés âgés », Norois [En ligne], 232 | 2014, mis en ligne le 30 octobre 2016, consulté le 13 janvier 2022. URL : http:// journals.openedition.org/norois/5161 ; DOI : https://doi.org/10.4000/norois.5161 


Presses
Universitaires
de Rennes
www.pur-editions.fr

\title{
Désacraliser la solidarité familiale Quand le cancer touche des immigrés âgés
}

\author{
Desacralisation of Family Solidarity. When Cancer Affects Elderly Immigrants
}

\begin{abstract}
Anaïk PiAN
Maître de conférences, géographe, Université de Strasbourg - Faculté de Sciences Sociales, DynamE UMR 7367 (anaikpian@yahoo.fr)

Résumé : À partir d’une enquête sociologique menée en région Bretagne, l'article éclaire la complexité des rapports sociaux qui mettent à l'épreuve la mobilisation des soutiens familiaux par les immigrés âgés atteints de cancer. Les rapports de place en jeu dans le système de parenté ainsi que les enjeux autour du rapport à l'information médicale feront l'objet d'une attention particulière. En conclusion, l'article invite à une réflexion plus générale quant à la manière de nommer la population concernée et, par là même, d'envisager le champ d'étude sur les immigrés âgés.
\end{abstract}

\begin{abstract}
Based on a sociological survey carried out in Brittany, this article highlights the complexity of social relationships that challenges the mobilisation of family support by elderly immigrants suffering from cancer. It will focus on the evolving relationships within the kinship system and on the relationship to medical information. In conclusion, the article introduces a more general reflection on naming the affected population and, therefore allows us to consider the field of study on elderly immigrants.
\end{abstract}

Mots clés : immigrés âgés - santé - cancer - famille - solidarité

Keywords: elderly immigrants - health - cancer - family - solidarity

\section{INTRODUCTION}

Le vieillissement des populations immigrées en France est une donnée qui a longtemps été occultée. La combinaison de plusieurs facteurs a contribué à laisser dans l'ombre cette problématique pourtant émergente : d'une part, la persistance d'un «mythe du retour » (Sayad, 1999) à l'égard des premières générations de travailleurs maghrébins et africains, venues prêter main-forte à l'économie française durant les Trente Glorieuses. Ce mythe a occulté le fait que le retour au pays d'origine, que ce soit en âge actif ou à la retraite, n'aura pas nécessairement lieu. D'autre part, les regards politiques et scientifiques se sont d'abord focalisés sur des catégories de populations plus visibles et médiatiques, faisant figure de problème social et politique, à l'image des jeunes « issus de l'immigration ». La thématique demeure ainsi, un temps durant, relativement marginale, contrastant en cela avec l'attention portée, au niveau national, au vieillissement général de la population (Temine, 2001). 
Au tournant des années 1970-1980, démographes, statisticiens et gériatres commencent toutefois à s'intéresser à la situation des immigrés vieillissants en France. En 1986, la revue Gérontologie consacre un numéro sur « La vieillesse des étrangers en France » en publiant les actes d'un colloque organisé, la même année, par des gérontologues avec le concours d'une association algérienne (Dubus et Braud, 2001). À partir de la fin des années 1990, la problématique des personnes immigrées âgées fait l'objet de numéros de revues spécialisées dans le champ des migrations (Migration Santé, 1999) et du vieillissement (Gérontologie et Société, 1999). Mais ce sont les années 2000 qui marquent véritablement l'essor de cette thématique et ce, à différents niveaux : à partir de cette période, aussi bien la littérature scientifique que les rapports publics et les enquêtes initiées par des institutions se multiplient sur le sujet (IGAS, 2002; HCI, 2005). Aujourd'hui, les personnes immigrées âgées constituent un groupe cible des actions menées par la DAAEN ${ }^{1}$ (Direction de l'accueil, de l'accompagnement des étrangers, de la nationalité) et le FAMI² (Fonds asile, migration, intégration). À l'échelle locale, y compris dans des régions de faible immigration historiquement, se multiplient les initiatives associatives donnant une visibilité aux difficultés d'accès aux droits sociaux (concernant le logement, la retraite, la santé...) des immigrés âgés. C'est le cas de l'Asalma (Association Santé migrants de Loire-Atlantique) à Nantes ${ }^{3}$ ou encore de l'UAIR (Union des associations interculturelles de Rennes) qui, depuis 2003, a développé plusieurs actions en faveur de cette population tout en sensibilisant les élus locaux à la question ${ }^{4}$.

L'intérêt croissant porté aux immigrés âgés s'appuie sur une réalité démographique de plus en plus marquée. Aujourd'hui, la France compte 6,7 mil-

1. La DAAEN a remplacé en 2013 la DAIC (Direction de l'accueil, de l'intégration et de la citoyenneté).

2. De même, le FAMI regroupe désormais le FER (Fond européen pour les réfugiés), le FEI (Fond européen d'intégration) et le FER (Fond européen pour le retour).

3. En 2005 et 2007, l'Asalma a réalisé deux études « Prise en compte des immigrés vieillissants » et "Les migrants âgés de la région nantaise au prisme du regard des professionnels ", dans le cadre du Programme régional d'insertion des populations immigrées des Pays de Loire (PRIPI).

4. Pour exemple, la journée organisée par l'UAIR à Rennes, le 25 octobre 2012, sur le thème « Accompagnement et prise en charge des personnes âgées immigrées ». Cette journée a été organisée avec le soutien de la Délégation Départementale de la Cohésion Sociale et de la Protection des Populations (DDCSPP), du Conseil général d'Ille-et-Vilaine, de la CARSAT, et de la Ville de Rennes. lions d'immigré-e-s (OMI, 2010 : 185). La population immigrée (hors Union européenne) de plus de 65 ans est d'environ 350000 personnes $^{5}$. Parmi elle, une majorité est originaire du Maghreb (210000) et d'Afrique subsaharienne $(30000)^{6}$, ce qui correspond aux grandes vagues d'immigration de travail des Trente Glorieuses. Comme le soulignent Attias-Donfut et al. (2006), l'âge moyen de la population immigrée a augmenté de plus de deux ans entre 1990 et 1999 (passant de 43,7 ans à 45,9 ans) tandis que le nombre des moins de 20 ans a diminué de $22 \%$. Bien que des nuances soient à relever selon les pays d'origine (ibid.), le vieillissement de la population immigrée résulte de la conjugaison de plusieurs phénomènes. Au vieillissement des « premières générations » ayant immigré au début de l'âge adulte s'ajoutent notamment les migrations tardives de « jeunes retraités ».

Au sein de ce numéro consacré au "mode de vie des aînés ", l'article a pour objet d'éclairer la manière dont des immigrés âgés atteints de cancer se positionnent, vivent et expérimentent les soutiens familiaux dans l'épreuve de la maladie. Si le vieillissement est souvent associé à la survenue de pluri pathologies (diabète, problèmes cardiaques, etc.) et à des limitations corporelles et incapacités diverses, ce sont la manière dont les liens familiaux sont sollicités dans la gestion de la trajectoire du cancer qui retiendra notre attention. La notion de trajectoire est entendue au sens où l'a définie Strauss (1992) dans ses travaux sur les maladies chroniques. Pour l'auteur, elle englobe l'ensemble du travail d'organisation du cours de la maladie effectué par les malades, leurs proches et les professionnels de santé, tout en tenant compte des conséquences de ce travail sur les différents acteurs en présence. Selon Strauss, cette gestion met le malade au défi de trois grands enjeux : outre la gestion de la maladie en elle-même, il s'agit de garder une maîtrise sur la vie quotidienne ainsi que sur sa biographie. La mobilisation des soutiens familiaux par les immigrés âgés atteints de cancer mais aussi, ce qui nous intéressera plus particulièrement ici, leurs diverses

\footnotetext{
5. Chiffres rappelés par Fatima Mezzouj (ministère de l'intérieur. Secrétariat général à l'immigration et à l'intégration) lors de la journée d'étude Le vieillissement des personnes immigrées âgées : l'accès aux droits, Résovilles, ASMALA (Association migrants Loire Atlantique), LABERS (Atelier de recherche sociologique), Université de Bretagne Occidentale, 23 novembre 2012 . 6. Idem.
} 
formes de mise à distance, participent de ces trois niveaux de gestion de la maladie.

L'article se structure en trois parties. La première resitue le cadre de l'étude en explicitant d'une part, le terrain d'enquête et d'autre part, le point de vue adopté - centré sur les soutiens familiaux. Les parties suivantes éclairent la complexité des rapports sociaux qui mettent à l'épreuve la mobilisation des supports familiaux par les immigrés âgés atteints de cancer, tout en donnant à voir les conséquences qui en découlent sur l'expérience de la maladie. Les rapports de place en jeu dans le système de parenté (seconde partie) ainsi que les enjeux autour du rapport à l'information médicale (troisième partie) feront l'objet d'une attention particulière. En conclusion, l'article questionne une dimension souvent considérée comme allant de soi et, de ce fait, rarement interrogée : sans prétendre clore le débat mais pointant plutôt sa complexité, l'ouverture proposée invite à une réflexion plus générale quant à la manière de nommer et catégoriser la population concernée et, par là même, d'envisager le champ d'étude sur les immigrés âgés.

\section{Cadre de L'Étude : une enquête SOCIOLOGIQUE AUPRÈS D'IMMIGRES ÂGES ATTEINTS DE CANCER EN BRETAGNE}

\section{Terrain d'étude et méthodologie}

L'enquête ${ }^{7}$ s'est déroulée dans trois villes bretonnes, Brest, Rennes et Quimper. En Bretagne, la population immigrée est peu importante, à la fois au sein de la région (en 2009, les immigrés y représentent $2,7 \%$ de la population) mais aussi par rapport aux autres régions françaises (Lardoux, 2012). La Bretagne arrive en tête des régions de l'Hexagone avec le taux d'immigration le plus faible, devançant en cela la Basse-Normandie et les Pays de la Loire

\footnotetext{
7. L'étude a été financée en 2010-2013 par l’Institut national du Cancer dans le cadre du programme Invuso : Trajectoires des malades, contextes de soin et inégalités sociales. Étude croisée des formes de vulnérabilité des patients atteints de cancer en évolution (axe 1, université de Rennes 2, Eso) et des patients immigrés et étrangers en Bretagne (axe 2, université de Bretagne Occidentale, Labers). Cet article repose sur les données d'enquête de l'axe 2. Il a également bénéficié des échanges dans le cadre du Programme MIGSAN (2010-2014) : Analyse des variations dans l'accès aux soins des populations migrantes à l'échelle locale (financement Inter-MSH - Bretagne, Pays de Loire, Poitou-Charentes - impliquant les universités de Rennes 2 (Eso), de Poitiers (Migrinter), d'université de Bretagne occidentale (Labers) ainsi que le Réseau-Ville-Hopital 35, devenu Centre Louis-Guilloux).
}

(Ibid.). La population immigrée est inégalement distribuée sur le territoire breton, les aires urbaines de Rennes, Brest et Lorient rassemblant à elles seules la moitié des immigrés (Dussud F.-X. et al., 2004) ${ }^{8}$. Elle est en outre moins âgée que l'ensemble de la population bretonne puisque $27 \%$ des immigrés de plus de 15 ans ont au moins 55 ans, contre environ $38 \%$ pour l'ensemble de la population (Lardoux, 2012). En chiffres absolus, 7000 immigrés de plus de 55 ans résident en Bretagne en 2011, contre, par exemple, 84000 en Provence-Alpes-Côte d'Azur, 93000 en Rhône-Alpes et 346000 en île de France $^{9}$. Par ailleurs, $43 \%$ des immigrés âgés de plus de 55 ans résidant en Bretagne en 2011 sont originaires du Maghreb et $14 \%$ d'entre eux sont originaires d'Afrique (hors Maghreb) ${ }^{10}$.

Au-delà de cette faible visibilité quantitative, le choix de la Bretagne comme terrain d'étude se justifie par plusieurs dimensions. Tout d'abord, l'intérêt d'un phénomène social ne se résume pas à son importance quantitative. Au contraire, le fait que les immigrés soient proportionnellement peu nombreux sur un territoire donné soulève des problématiques particulières au niveau de l'accès aux soins d'une partie de cette population, des logiques d'adressage entre professionnels, etc. (Pian, 2012). Ensuite, comme il a été précédemment souligné, dans certaines villes bretonnes, à Rennes mais aussi à Brest par exemple des initiatives associatives mobilisant le soutien d'élus ou appelant à leur sensibilisation, se sont progressivement mises en place ou sont en cours d'élaboration, afin d'accompagner les immigrés âgés dans leurs démarches de soin. Même s’il n'est pas possible de détailler ces initiatives dans le cadre de cet article, celles-ci viennent suppléer, remplacer ou compléter d'éventuels supports familiaux. Elles témoignent par là même des limites de ces derniers, qu'ils soient inexistants, trop faibles ou peu adaptés à des contextes de relations donnés.

Concernant la population d'étude, deux catégories d'immigrés âgés atteints de cancer sont ici prises en compte. La première regroupe des immigrés venus en France dans les contextes d'immigration de travail d'après-guerre et post-colonial. La seconde,

\footnotetext{
8. Sur l'histoire de l'immigration en Bretagne, cf. Morillon et al., (2007).

9. Source : Secrétariat général à l'immigration et à l'intégration, cité in Laacher (2014:61). Ces chiffres incluent l'ensemble des immigrés, tous pays d'origine confondus.

10. Source : Secrétariat général à l'immigration et à l'intégration, cité in Laacher (2014: 62).
} 
particulièrement invisibilisée dans la littérature, concerne des femmes (dans le cas présent veuves ou divorcées), nouvellement arrivées en France à un âge avancé, et initialement venues pour aider leur fille ou fils à élever leurs enfants, souvent suite à un veuvage ou un divorce. Nous nous centrerons dans le cadre de cet article sur la population originaire du Maghreb et d'Afrique subsaharienne, celle-ci constituant la majeure partie de notre corpus.

Au niveau méthodologique, l'enquête relève d'une approche qualitative. Elle repose d'une part, sur quelques observations réalisées dans un établissement de lutte contre le cancer et d'autre part, sur des entretiens réalisés auprès de patients, de proches et de professionnels de santé et du secteur social $(\mathrm{N}=81)$. Les immigrés âgés correspondant au profil de population ici retenu ne représentent qu'une partie (une dizaine) du corpus des patients rencontrés. Ils ont été rencontrés par le biais de services hospitaliers, de réseaux de cancérologie ou de soins palliatifs ainsi que des associations interculturelles. Bien qu'ils ne constituent pas un échantillon représentatif sur le plan statistique, les entretiens menés permettent de restituer des logiques d'action et de sens montrant en quoi la mobilisation de supports familiaux n'a rien de «naturelle » et n'est pas exempte de tensions ${ }^{11}$. Les entretiens avec les immigrés âgés se sont déroulés à domicile, dans des locaux associatifs ou au sein des services de soin. Nous avons privilégié des entretiens réitérés dans le temps lorsque l'état de santé des intéressés le permettait.

\section{Pourquoi s'intéresser aux soutiens familiaux dans l'expérience du cancer?}

Comme le rappellent Attias-Donfut et Delcroix (2004), la majorité des immigrés âgés vit aujourd'hui en famille, quand bien même la figure du retraité isolé vivant en foyer est la plus médiatique. Or, la dimension familiale demeure relativement peu explorée dans les travaux portant sur la santé des immigrés âgés. Les études existantes mettent plus l'accent sur les conditions et difficultés d'accès aux soins de ces populations (Math, 2014), leur état de santé objectif et subjectif (Attias-Donfut et al., 2006) ou encore sur leur difficulté à faire recon-

11. Par souci d'anonymat, tous les noms ont été modifiés. naître des maladies professionnelles - par exemple dans le cas d'exposition à des produits cancérigènes (Boujasson, 2009).

Certes, il ne s'agit pas de dire que la dimension familiale est absente de la littérature consacrée aux immigrés âgés. Elle s'y retrouve même de manière transversale. On peut prendre pour exemple le débat moral et politique, relayé par plusieurs études, sur la légitimité ou non de la vieillesse immigrée. Cette légitimité est très souvent discutée à partir du rapport au travail qui, au départ, légitimait et justifiait, tant vis-à-vis des pays d'origine que d'accueil, la venue des immigrés sur le territoire français. La vieillesse illégitime (Sayad, 1999) est celle des corps qui, ne remplissant plus leur fonction de force de travail, perdent leur raison d'être en immigration/ émigration même si, à l'âge de la retraite, continuer d'envoyer de l'argent à la famille au pays d'origine permet de justifier le non retour. Plusieurs années plus tard, d'autres auteurs (Attias-Donfut et al., 2006) défendent au contraire l'idée d'une « vieillesse enracinée ", eu égard aux conditions de vie des intéressés, de la manière dont ils se positionnent par rapport au mode de vie de leur société d'origine et de leurs attaches familiales en France (présence d'enfants, de petits-enfants, etc.). Dans ce prolongement, les études interrogeant le rapport à la mort des immigrés âgés (Barou et Gallou, 2011) conduisent également à s'intéresser à la mémoire familiale et aux rapports inter-générationnels par-delà les territoires, dès lors que le lieu et les modalités de l'inhumation recèlent de forts enjeux symboliques et identitaires. Mais ce sont aussi les travaux consacrés aux conditions d'habitat des immigrés retraités dans les foyers Sonacotra puis Adoma ${ }^{12}$ qui abordent, en creux, la question des liens familiaux. Et ce, qu'il s'agisse d'évoquer la double désaffiliation de ces résidents - par rapport aux pays d'accueil et d'origine - (Hmed, 2006) ou les formes de sociabilités contractées entre eux (Meslin, 2010), pansant un fort sentiment de solitude.

Bref, si la question des rapports familiaux revient en filigrane de plusieurs thématiques afférentes au champ d'étude consacré aux immigrés âgés, il reste qu'une zone d'ombre demeure quant à la manière dont, en contexte de maladie grave, se régulent, se négocient, sont mobilisés ou non, des soutiens fami-

12. En 2007, la Sonacotra change de nom pour Adoma. 
liaux. Or, des travaux ont souligné l'importance du rôle de l'entourage du malade dans les soins oncologiques ainsi que les attentes très normées des soignants à l'égard des proches (Marche, 2014).

Outre une complémentarité avec la littérature existante, le second intérêt d'une focale sur les supports familiaux se situe en rupture avec des stéréotypes relativement fréquents à l'égard des familles maghrébines (et, dans une moindre mesure, subsahariennes). Ces représentations se retrouvent, comme nous avons pu le constater lors de notre terrain, parmi les soignants prenant en charge cette population. Alimentées par des anecdotes ou des expériences singulières, elles tendent à être généralisées de manière englobante et homogène. Liens fusionnels entre les mères maghrébines hospitalisées et leurs filles qui téléphonent sans cesse dans le service; place prégnante des proches qui souhaitent faire eux-mêmes la toilette de leur parent ou conjoint hospitalisé à la place des aides-soignantes ; présence continue de la famille se relayant auprès du parent hospitalisé et apportant de la nourriture (même si cela transgresse les régimes prescrits par les médecins), etc. Cette présence familiale, dès lors qu'elle est considérée comme excessive voire « envahissante » par les professionnels, est perçue comme entravant le bon fonctionnement du service, nuisant à l'autonomie du patient ou encore dépossédant les soignants ou personnel hospitalier du volet relationnel de la prise en charge - volet particulièrement valorisé par les aides-soignantes et les agents hospitaliers rencontrés, qui y trouvent une valorisation de leur identité professionnelle en marge du « saleboulot» (Arborio, 1995) effectué. Mais la famille fait-elle toujours «bloc » derrière le parent malade et ce dernier mobilise-t-il toujours les supports familiaux disponibles?

\section{DES RAPPORTS DE PLACE EN JEU DANS LE SYSTÈME DE PARENTE}

La mobilisation des supports familiaux par les immigrés âgés atteints de cancer constitue certes, une ressource pour communiquer avec les médecins, organiser les prises de rendez-vous, aider aux actes de la vie quotidienne, etc., mais peut aussi revêtir un coût social symbolique. Ce coût se donne à lire dans plusieurs situations de cohabitation intergénérationnelle observées. Il est également marqué lorsque l'appel à un soutien familial contribue à renforcer une inégalité de rapports de place au sein de l'espace social et/ou du groupe familial. Ces limites dans l'accompagnement face à la maladie s'expriment particulièrement à travers le modelage de trois types de soutien : le soutien inversé, le soutien contenu, le soutien contraint et contraignant.

\section{Être malade sans avoir de chez soi}

Être malade «sans avoir de chez soi » est une situation dans laquelle se retrouvent certains immigrés âgés atteints de cancer. C'est notamment le cas pour des personnes retournées vivre dans leur pays d'origine à l'âge de la retraite, mais ayant gardé une domiciliation en France - chez un tiers - où elles se rendent régulièrement pour ne pas perdre leurs droits sociaux afférés au séjour. Les circulations migratoires sous forme de va-et-vient entre pays d'accueil et d'origine sont en effet fréquentes parmi les immigrés retraités (Attias-Donffut et al., 2006). Lors de leur venue en France, il n'est pas rare qu'ils se prêtent à des examens de santé. Lorsqu'un cancer est diagnostiqué, l'annonce de la maladie met fin de manière brutale aux allers-retours et implique de rester sur le territoire français, pour une durée incertaine et imprévue. Lorsque les intéressés ne sont pas hospitalisés en établissement ou qu'ils le sont ponctuellement (hôpital de semaine par exemple), ils résident au domicile de tiers, en l'occurrence leurs enfants adultes parmi les cas rencontrés. L'hébergement par un descendant est également fréquent parmi les femmes immigrées arrivées en France à un âge avancé afin de suppléer leurs enfants dans l'éducation des petits-enfants. AttiasDonfut et Gallou (2006) soulignent qu'en fonction des pays d'origine, des trajectoires migratoires et des référents culturels, les cohabitations avec un parent âgé dépendant s'inscrivent dans des normes et valeurs plus ou moins partagées. Mais au-delà de ces normes et valeurs telles qu'elles sont énoncées, comment est vécu, par les ascendants atteints de cancer, le partage de l'espace - et de son intimité?

Tout d'abord, les conditions de logement ne sont pas nécessairement adaptées à l'accueil sur la durée d'un parent malade (manque de chambre...). Ne pas déranger est une préoccupation récurrente des immigrés âgés, notamment parmi ceux ayant une domiciliation administrative chez leurs enfants. 
C'est pourquoi lorsque plusieurs enfants résident dans la même ville, certains choisissent de résider en alternance chez ces derniers. Cette alternance peut aussi répondre à des préoccupations organisationnelles. Abdelkrim (81 ans), originaire du Maroc, est arrivé en France dans les années 1960. Durant toute sa carrière, il travaille comme ouvrier dans le secteur automobile en région parisienne. Sa femme et ses enfants restent vivre au Maroc, où il retourne périodiquement le temps des vacances ou de latence entre deux contrats. À sa retraite au début des années 1990, il repart s’installer au Maroc, mais revient régulièrement en France, notamment en Bretagne, où deux de ses enfants résident désormais (sa fille, suite à son mariage; son fils, initialement dans le cadre d'études). En 2011, suite à des douleurs inexpliquées et une anémie persistantes, il décide de réaliser des examens médicaux en France. Un cancer du côlon à un stade très avancé est diagnostiqué. Il reste alors suivre un traitement en Bretagne, où il loge alternativement chez sa fille (devenue assistante maternelle, 35 ans) et son fils (au RSA, 30 ans). Au fil des semaines, l'arrangement suivant s'est mis en place : la veille des séances de chimiothérapie, il dort chez son fils qui habite plus près de l'hôpital et qui l'accompagne lors des consultations. Le reste de la semaine, il est hébergé chez sa fille qui veille sur lui entre le passage des infirmières à domicile. Si cette répartition répond à des considérations pragmatiques liées à la distance au lieu de soins, on ne peut s'empêcher de constater qu'elle correspond également à une division sexuée du soutien profane entre ascendants ${ }^{13}$.

Être malade sans avoir de chez soi peut impliquer un effort particulier de gestion du stigmate qui rappelle, par là même, la légitimité fragile de la présence de « l'invité ». Il s'agit alors de maîtriser les bruits et les odeurs liés aux effets secondaires des traitements (vomissements, diarrhées, fuites urinaires, etc.), mais aussi de maîtriser ses émotions (ne pas pleurer, notamment devant les petitsenfants). S'efforcer de se lever pour aller à table même sans appétit; participer - notamment pour

13. D’une manière générale, plusieurs travaux (Gucher, 2010) ont montré que le soutien aux parents âgés tend à se décliner différemment selon les ascendants filles et garçons, les seconds s'occupant plus des questions administratives, les premières des tâches relevant des activités de soin au corps, dans la continuité d'une socialisation sexuée faisant passer comme «naturelles » chez les femmes, des dispositions ou qualités de « nursing » pourtant socialement construites. les femmes - à quelques tâches ménagères, sont des pratiques récurrentes qui soulignent, par ailleurs, des inégalités de genre dans les rôles sociaux à tenir y compris dans l'expérience de la maladie.

En témoigne la manière dont Françoise, Guinéenne âgée de 65 ans, évoque son quotidien au domicile de sa fille, Commerçante, Françoise est venue plusieurs fois en France, avant de venir s'y installer au milieu des années 2000, pour prêter main-forte à sa fille (vendeuse, 36 ans), mère de quatre enfants, devenue veuve. Elle s'est alors installée chez elle, d'abord avec « entrain » dit-elle car elle pouvait alors apporter tout son soutien à sa fille. Mais, depuis qu'elle est atteinte de cancer, elle trouve la cohabitation très difficile à vivre :

«Il y a les enfants, alors ce n'est pas facile... Quand j'ai commencé à perdre les cheveux. Je me suis dit: "je vais mettre un foulard" et puis, petit à petit, il faisait chand, donc je suis restée complètement sans foulard... Ils ont demandé pourquoi je n'avais plus de cheveux, alors leur maman leur a dit que j'étais malade... [...]. Même quand on accepte les choses, il y a des moments de blues, quoi. [...] Parce que je me suis rendu compte après la chimio, qu'on est très malade. En fait, pendant six jours, j'étais fatiguée, j'avais des nausées, des fièvres. Et ça vous oblige pratiquement à ne pas avoir d'idées positives. »

Et vous échangez avec votre fille?

"Je préfère me réserver, je ne veux pas trop l'embêter. Parce que ma présence, est déjà suffisante [...]. Je préfère être dans mon coin et supporter certaines choses parce que je ne veux pas qu'on se dise: "elle exagère, elle peut supporter bien." Vous savez les gens, ils ne comprennent pas. À ce stade de la maladie, je ne comprenais plus, je pensais qu'on fait semblant. Quand vous dites que vous avez mal, c'est que vous avez vraiment mal. Vous ne voulez embêter personne. On a tendance à se renfermer à ce stade de douleur [...]. Et j'essaye de montrer un visage en sourire, parce que je ne veux pas qu'on se lasse de moi [...]. Et bon, j'essaye de manger à table même quand je n'ai pas envie. Parce qu'il y a des moments, vous savez, les effets secondaires se font sentir avec un dégoût pour la nourriture... Donc même si je n'ai pas envie de manger, je m'efforce d'être à table avec les enfants. À chaque fois qu'il y a un repas, 
je m'efforce à débarrasser, et je m'efforce à faire la vaisselle. Même si quelques fois, c'est douloureux, ça fait mal parfois, de mettre les mains dans l'eau. »

Pour les grands-mères arrivées en France à un âge avancé, le cancer est d'autant plus difficilement vécu qu'il implique des formes de soutien inversé par rapport au projet migratoire. Non seulement elles ne peuvent plus satisfaire aux activités du care ${ }^{14}$ liées à leur grand-parentalité, mais elles deviennent destinataires de formes de soutien qui impliquent souvent pour le descendant une réorganisation du quotidien (garde des enfants, réaménagement des horaires du travail salarié, voire déménagement pour se rapprocher des lieux de soin, etc.).

La cohabitation intergénérationnelle ne prémunit pas nécessairement, par ailleurs, contre le sentiment de solitude : la proximité spatiale avec les proches peut s'accompagner d'une distance sociale dans le partage des émotions, le parent malade ne souhaitant pas constituer un «fardeau » supplémentaire. Mais la figure du «fardeau » n'est pas seule en jeu : s'y adjoint, comme le donne à voir le discours de Françoise, celle de l'indicible. Il en résulte des formes de soutien contenu ou d'autocensure, où le parent malade tente de limiter les sollicitations envers ses proches. De même, les ascendants sont plusieurs à rapporter que la présence à domicile de leur parent malade ne s'accompagne pas, pour autant, de véritables échanges sur la maladie qui reste "quelque chose dont on ne parle pas beaucoup ». L'expérience de la solitude est d'autant plus accentuée que les intéressés entretiennent peu de relations en dehors du cercle familial : il en est ainsi pour des travailleurs immigrés retraités dont les enfants résident dans une autre ville que celle où eux-mêmes ont vécu. Mais c'est aussi le cas pour des femmes immigrées arrivées à un âge avancé en France et dont l'espace de sociabilité est resté cantonné à l'espace du foyer. Les visites reçues sont, le cas échéant, celles d'amis de leurs enfants et, bien qu'elles permettent de «casser » le rythme de la journée, elles marquent un décalage entre générations : il ne s'agit pas de leurs propres amis, ni mêmes de personnes du même âge qu'elles. L'on pourrait ainsi parler d'une forme de solitude géné-

14. Le terme se réfère aux « soins coutumiers liés aux fonctions d'entretien de la vie » (Cresson, 2006) mais reste difficilement objectivable. Il est proche du "prendre soin " même si les frontières des deux notions ne se superposent pas toujours. rationnelle où la convivialité et la forte présence de l'entourage élargi au pays d'origine fait défaut.

À un autre niveau encore, la mobilisation des supports familiaux est susceptible de prendre une forme que l'on peut qualifier de contrainte et contraignante, prolongeant ou accentuant des rapports de domination au sein de la sphère familiale.

\section{Soutiens versus rapports de domination}

Pour rendre compte de cette configuration, nous partirons de la situation de Beija. Son parcours fait partie de ces migrations longtemps invisibilisées dans la littérature : celles de femmes ayant migré seules, en dehors du regroupement familial ou du sillage tracé par leurs conjoints (Moujoud, 2003).

Beija est une Marocaine de plus d'une soixantaine d'années, atteinte d'un cancer de l'utérus. Arrivée en France à 28 ans, elle y rejoint son frère aîné (ingénieur), qui vient d'avoir un enfant. Au Maroc, où elle n'a pas été scolarisée, elle a été mariée par son père puis découvre que son époux avait déjà une première femme, qui ne pouvait lui donner d'enfants. Elle aura deux enfants avec son mari puis, souffrant de dépression, part s'installer seule en France où elle rejoint son frère. Quelque temps plus tard, elle trouve un emploi de cuisinière. Aujourd'hui, Beija parle bien le français (du moins en ce qui concerne les conversations courantes), mais ne sait ni le lire ni l'écrire. En 2011, alors qu'elle est en vacances au Maroc, elle est prise de saignements vaginaux importants, qu'elle attribue dans un premier temps à une « reprise de règles ». À son retour en France, elle prend rendez-vous avec son médecin généraliste, car commençant à s'inquiéter de ces pertes de sang persistantes. C'est alors qu'un cancer de l'utérus à un stade avancé est diagnostiqué. En 2012, alors qu'elle est hospitalisée, l'équipe médicale propose de lui mettre une poche urinaire tout en précisant que son cancer restera incurable. N'étant pas sûre d'avoir bien compris, Beija fait appel à son frère pour qu'il soit présent lors de la prochaine rencontre avec le médecin. Elle s'oppose finalement à l'acte, craignant les effets de la stigmatisation sans pour autant y voir des bénéfices en termes de guérison. Son frère insiste pour la convaincre. Beija fait part de son soulagement lorsque le médecin remet son frère à sa juste place, lui rappelant que la décision thérapeutique revient au patient : 
"Le docteur a dit: "Monsieur, je ne peux pas le faire si elle n'en a pas envie. C'est elle, c'est son corps, c'est ni vous ni moi." Voilà, j'ai dit: "Il y en a un qui a compris." Mon frère, maintenant, il est contrarié, il est contrarié, parce que je ne veux pas le faire. Je dis : "non, c'est pas toi qui es à ma place." "

L'intervention du frère peut être perçue, à première vue, comme une ressource potentielle pour Beija qui a du mal à se repérer au sein de l'univers médical. Mais le fait que ce dernier tente d'imposer son point de vue sur une décision concernant son propre corps, qui plus est une partie intime, est vécu comme une violence symbolique. Lincident contribue à accentuer des relations familiales tendues depuis le divorce puis le remariage de son frère. Par la suite, c'est sous la forme d'un soutien contraint et contraignant, à défaut d'autres alternatives, que Beija fera appel à ce dernier. Ce soutien semble lui-même parfois difficilement concédé par son frère : ainsi, elle raconte comment ce dernier s'oppose à ce que sa femme l'aide dans les tâches ménagères, activités qu'il percevrait comme ne relevant pas de son rang social.

«Il est tellement fier, c'est sa femme, il ne veut pas qu'elle aille faire la boniche chez les autres. Mais je ne suis pas les autres. S'ils ont besoin de quelque chose, je suis là la première, même j'y vais à quatre pattes [...]. Mon frère [...] a un beau terrain, en face de la plage. Il a une belle maison. Mais moi je n'ai rien, mais j’ai ma fierté aussi, hein. Moi aussi, j'ai travaillé toute ma vie, je n'ai jamais rien demandé. »

Depuis sa maladie, à défaut de pouvoir satisfaire à l'entretien collectif de l'immeuble où elle est locataire, elle verse une c ontribution financière réclamée par le syndic. À la sortie de l'une de ses hospitalisations, son frère a proposé de l'héberger, afin qu'elle ne reste pas seule à domicile. Deux semaines plus tard, Beija préfère rentrer chez elle : en larmes, elle raconte les soupirs et remarques à mi-voix de sa belle-sœur, peu encline à jouer le rôle de garde malade. "Au moins, chez moi, je suis toute seule et ne dérange personne », souligne-t-elle, en insistant sur le fait que, toute sa vie durant, elle a appris à se débrouiller seule.

Comme l'a montré Metboul (2010) à partir d'une enquête dans des services hospitaliers en Algérie, la mobilisation des soutiens familiaux peut aussi renforcer des rapports de domination liés à un ordre patriarcal et/ou à la position occupée dans la hiérarchie sociale. Derrière les discours conventionnels sur l'entraide familiale très souvent sacralisée voire «naturalisée » (ibid.) dans les familles maghrébines, se dessinent des pratiques beaucoup plus nuancées qui peuvent renforcer des rapports inégalitaires de genre, de classe, de génération (ou de fratrie/sororie) au sein de l'institution familiale. La remarque de Benveniste (2002) à propos des communautés, selon laquelle celles-ci n’impliquent pas nécessairement un milieu social unifié, mais que c'est souvent le rapport à l'extérieur et - ajouterons-nous, le regard porté de l'extérieur - qui alimente cette homogénéisation, trouve un écho à propos des mises en acte des soutiens familiaux.

Cette mise en forme est particulièrement sensible dans le rapport à l'information médicale. Maladie à caractère létal où les diagnostics se précisent et évoluent dans le temps, où les pronostics reposent sur des catégories probabilistes (Ménoret, 1999) et où les traitements sont hautement spécialisés, le cancer implique des enjeux de vie et de mort. Dans ce contexte d'incertitude, les enjeux de voilement/ dévoilement de la maladie influent sur la mobilisation des soutiens familiaux par les immigrés âgés.

\section{LES SOUTIENS FAMILIAUX À L'ÉPREUVE DES LOGIQUES DE VOILEMENT DE LA MALADIE}

Les personnes immigrées âgées ne parlant pas ou peu français sont souvent accompagnées dans les services de soin par des proches endossant le rôle d'interprète. Ce recours aux membres de la parenté est, pour des raisons organisationnelles et financières, largement utilisé dans les établissements. Les proches sont aussi parfois considérés de plus grande confiance qu'un interprète extérieur par leur parent malade. Leur présence les rassure, permettant de revenir de manière différée sur les propos des soignants ${ }^{15}$. Cependant, ce recours a aussi ses revers ou du moins ses limites. Outre des questions liées

15. Selon les situations, ces derniers perçoivent de manière ambivalente la présence des proches endossant le rôle d'interprète. Si les professionnels de santé peuvent voir dans les membres de l'entourage des alliés potentiels pour l'éducation (ou alliance) thérapeutique des patients ([ré] explications des effets secondaires des traitements au patient, surveillance des symptômes, etc.), il reste que le risque de travestissement ou de voilement de l'information concernant les diagnostics et pronostics est une préoccupation majeure des équipes médicales. Sur ce sujet, cf. Pian (2014). 
à la pudeur et l'exposition inévitable d'une partie de leur intimité, tel qu'il le sera détaillé plus loin, les malades peuvent chercher à limiter l'implication du proche afin qu'il continue à vivre le plus normalement possible (poursuite des études, loisirs, sorties, engagement dans leur propre vie familiale). Et ce, d'autant plus lorsque le contexte familial est tel que l'hospitalisation pour cancer implique le report à la charge de l'entourage de diverses tâches domestiques et du care. Sur un autre registre, c'est aussi le sentiment d'une mise à l'écart voire d'exclusion dans les échanges qui, en situation d'interprétariat, peut être mal vécu. Des immigrés âgés parlant et comprenant le français bien que de manière limitée, expliquent se sentir dépossédés de l'interaction avec le médecin en présence du proche. Lorsque la consultation se déroule sans intermédiaire, les praticiens sont contraints de parler lentement, parfois à l'aide de gestes, pour qu'une communication minimale puisse s'établir. En présence du proche en revanche, les médecins parlent beaucoup plus vite ce qui rend plus difficile pour les intéressés une compréhension immédiate et une maîtrise - même minimale - de la situation. Le jeu de regards se déplace également : des malades regrettent que les praticiens ne les regardent « même plus » et font comme «s'ils n'étaient pas là ». Certains dénoncent le fait que ces derniers, alors incapables de les reconnaître, pourraient se tromper de patients. Il en découle un sentiment de réification où le parent malade a l'impression d'être objet et non sujet de l'échange.

Mais les modalités de communication ne se limitent pas aux échanges verbaux : le rapport à l'écrit a également son importance et peut être problématique pour les immigrés âgés ne sachant ni lire ni écrire. Il peut même les mettre particulièrement mal à l'aise - dans des contextes ordinaires ou dans le monde médical - dès lors que leur maniement de la langue française à l'oral, ne laisse pas nécessairement soupçonner à leur interlocuteur qu'ils ne sont en mesure de remplir un formulaire ou lire une plaquette d'information. C'est dans une situation similaire que s'est trouvé Jalil (73 ans), ouvrier retraité d'origine algérienne : parlant bien français, il n'a jamais appris à le lire ni l'écrire, lui qui, par ailleurs, a arrêté très tôt les bans de l'école en Algérie.

Originaire de la région de Constantine, Jalil arrive en Bretagne en 1963, où il rejoint plusieurs amis venant de s'y installer. Il travaille deux ans dans le bâtiment puis, après un an sans activité, il trouve un emploi d'éboueur. Jusqu'à sa retraite prise en 1999, il occupe ce métier. Quelque temps après son installation en France, il rencontre une Française avec qui il aura trois enfants, en union libre. Le couple se sépare six ans plus tard. Jalil cohabite alors avec une autre Françoise et de leur union naît un autre enfant. Après cinq/six ans de vie commune, le couple se sépare à nouveau. En 1979, Jalil fait venir par le biais du regroupement familial son épouse résidant en Algérie, ainsi que ses deux enfants nés au pays, âgés entre 3 et 8 ans. En effet, il s'était marié à Constantine alors qu'il était âgé d'une vingtaine d'années, juste avant sa venue en France. Sa femme, elle, n'avait alors que 14 ans. Après que son épouse l'ait rejoint en Bretagne, le couple a quatre autres enfants, deux filles et deux garçons. Depuis maintenant plusieurs années, Jalil n'a plus de contacts avec les enfants de ses premières conjointes. C'est en 2006 qu'il apprend être atteint d'un cancer de la prostate. Suite à l'apparition des premiers symptômes (envie très fréquente d'uriner, douleurs), il met près d'un an à consulter son médecin généraliste qui l'envoie alors effectuer des analyses et prélèvements d'urine. Les résultats confirment l'existence d'un cancer et Jalil, réorienté vers l'hôpital, est opéré de la prostate. Même s'il aurait aimé connaître le contenu des plaquettes d'information sur la maladie et les traitements remises par les professionnels de santé, il explique comment il les jetait systématiquement à la poubelle : documents déchirés et abandonnés parmi les déchets alors que, souligne-t-il avec dépit, il aurait souhaité pouvoir déchiffrer entre les lignes. Ne sachant lire, il ne voulait pas, cependant, demander à ses enfants de lui faire la lecture des documents transmis et ce, malgré des ressources familiales présentes : qu'il s'agisse de ses deux plus jeunes fils, vivant encore au domicile parental ou de ses autres enfants, vivant dans la même ville. De même, il était inimaginable pour lui de s'en remettre à un voisin ou un ami. Le contrôle de l'information sur soi se fait ainsi au détriment de la quête d'information ${ }^{16}$. Cette mise à distance des ressources familiales s'inscrit plus précisément dans plusieurs registres de sens.

16. Bien que ce point ne puisse être développé ici, le médecin généraliste incarnant la figure du médecin de famille peut constituer un interlocuteur privilégié, faisant l'interface entre le milieu hospitalier et les patients immigrés âgés. 
Tout d'abord, solliciter ses enfants aurait contribué à altérer la figure de l'autorité paternelle revendiquée. Dans son récit, l'expérience subjective est celle de la gêne, voire de honte : si la honte peut être entendue comme un « ensemble hétérogène d'attitudes mentales qui ont en commun une dépréciation de soi »(Fassin et Morice, 1997 : 296), elle s'inscrit plus largement dans une «morale sociale, une morale du regard des autres » (De Sardan, 1996 : 110) agissant comme un moyen de régulation sociale (Ibid.). Ici, s'expriment la gêne et la honte d'être incapable de se débrouiller seul; mais aussi la gêne, la honte, d'exposer publiquement l'intimité d'un corps malade et défaillant, touchant à une intimité la plus profonde. Jalil vit très mal les limitations sexuelles faisant suite à son opération, altérant le rapport à soi et aux autres. Depuis son cancer, il explique « ne plus pouvoir faire l'amour avec sa femme » et se qualifie de «mort-vivant ». La révélation d'une virilité défaillante marque une rupture brutale avec un corps subitement perçu comme "vieux », et devenant à la fois étrange et étranger ${ }^{17}$.

Ne pas «perdre la face », du moins totalement, explique pour partie la mise à distance des ressources familiales pourtant potentiellement disponibles. À cet égard, il était particulièrement impensable pour Jalil de solliciter sa fille cadette, infirmière, afin de lui demander des compléments d'information sur la maladie et les traitements. Comme pour d'autres immigrés âgés rencontrés, la mise à distance de proches engagés dans des carrières professionnelles soignantes répond à la volonté de préserver l'information sur soi face à un entourage qui pourrait comprendre, savoir, ou anticiper trop choses. Cette mise en réserve vise autant à ne pas inquiéter les proches qu'à ne pas trop se dévoiler pour garder la tête haute. Réserve qui, toutefois, ne saurait se réduire à une détermination culturelle, selon une conception essentialisée de la culture : certes, le cancer est un mot plus ou moins tabou qui, selon les cultures, se dit ou non, ou d'une manière détournée, distanciée, d'autant plus lorsqu'il touche des parties intimes. Au Maroc, le cancer est parfois désigné sous la métaphore de la «mauvaise maladie». Mais c'est bien en contexte, selon les situations, que sont mis en avant, négociés et réinterprétés des modèles culturels sur le dire ou

17. Sur le rapport au corps dans l'expérience de la maladie et du vieillir, cf. Marche et Pian (2014). non ou encore sur la manière de dire : ainsi, si la fille d'une patiente algérienne âgée trouvait important de révéler le diagnostic du cancer à sa mère, elle approuve en revanche le fait que sa tante, plus jeune, et au même moment atteinte d'un cancer en Algérie, n'ait pas été informée du nom de sa maladie : d'une part, en raison de son âge, celle-ci étant de plusieurs années plus jeune que sa mère; d'autre part, en raison du contexte de soin, beaucoup moins performant en Algérie selon elle.

Enfin, dans le cas de Jalil, une autre dimension liée aux représentations et causalités du cancer interfère dans la mise à distance des supports familiaux. En effet, celui-ci se demande si le cancer n'est pas dû aux transgressions des préceptes musulmans qui ont ponctué sa jeunesse : concubinage et enfants hors mariage, adultère, consommation de porc et d'alcool... Parler de la maladie avec ses proches c'est rendre visible et rappeler au grand jour la « carrière déviante » qui a marqué son parcours de vie; carrière à laquelle il estime avoir progressivement mis fin suite à la venue en France de son épouse algérienne.

Il est par ailleurs fréquent que la mise à distance des supports familiaux dans la quête d'informations alimente une sorte de paradoxe : alors que cette mise à distance répond à la volonté de garder une maîtrise sur sa trajectoire de maladie, notamment au niveau du travail biographique, le manque d'information ressenti peut concourir à produire, du moins en partie, un effet inverse : l'impression de ne pas avoir d'emprise sur la gestion de la maladie ni ses effets secondaires, ayant des conséquences dans la vie quotidienne.

Si le rapport à l'information médicale - et ce qu'il implique en termes de voilement/dévoilement de la maladie - est une dimension centrale dans les logiques de mobilisation des supports familiaux par les immigrés âgés, il l'est aussi dans la manière dont les professionnels de santé jugent et qualifient la «bonne distance » tenue par les proches des patients à l'égard de leur parent ${ }^{18}$.

18. Dans cette définition de la «bonne distance " qui, toutefois, fluctue selon le type de service (par exemple entre un service de soins palliatifs et un hôpital de jour) et les moments de la trajectoire du cancer, tendent à être mis en opposition par les soignants deux types d'autonomie tels que le formule Fan (1997). Selon l'auteur, en occident, le principe d'autonomie (tel qu'il est «idéalement » énoncé du moins) donne priorité à l'auto-détermination du patient (self-determination), considéré comme un acteur éclairé de sa santé selon une conception d'indépendance individuelle, au sein d'un modèle médical qui, dans les codes déontologiques 


\section{Conclusion}

Les données d'enquête conduisent à déconstruire les visions culturalistes voire essentialistes tendant à naturaliser l'entraide familiale dans les familles maghrébines et subsahariennes, où le « tout communautaire » anesthésierait toute forme de tension intra-familiale ${ }^{19}$. Sans pour autant signifier que dans ces familles la solidarité n'existe pas, il importe de souligner combien celle-ci engage des rapports sociaux complexes, souvent invisibilisés et qui, au-delà de représentations culturelles, ne peuvent être saisis sans une prise en compte longitudinale des parcours de vie. Deux précisions méritent alors d'être apportées.

Tout d'abord, les logiques de mise à distance décrites ne doivent pas être envisagées de manière statique mais dynamique. Pouvant évoluer et se combiner dans le temps et l'espace des trajectoires de maladie, elles invitent à distinguer soutiens potentiels et effectifs ainsi qu'à resituer les supports mobilisés dans l'articulation des rapports de classe, de sexe, d'âge et de relations inter-ethniques.

Ensuite, il convient de rappeler que la sacralisation de la solidarité familiale n'est pas une exclusivité portée à l'encontre des seules familles composées d'immigrés - en témoigne les débats autour de la notion «d'aidant naturel » (Blanc, 2010) - même si, à l'égard des premiers, elle est particulièrement marquée. La perspective comparative offre ainsi une perspective intéressante dès lors qu'elle permet de saisir « la dialectique entre l'universel et le particulier $($ Green, $2002: 7)$. Plus largement, importe-

et la législation, porte globalement l'obligation d'une information claire et «loyale » au patient. Dans les pays d'Asie du Sud est en revanche, domine un principe d'autonomie familiale (familiy-determination), encastré dans un cadre communautaire, où le noyau familial détient une autorité première sur les décisions médicales. Ce sont les représentants familiaux qui, en premier lieu, sont tenus d'être informés de la maladie grave ou du pronostic pour ensuite décider de le divulguer ou non au parent malade. Dans les représentations des équipes médicales, les patients et leurs proches d'origine maghrébine, africaine ou des pays de l'ex-bloc soviétique sont également perçus comme rattachés à un modèle d'autonomie familiale. Toutefois, en rupture avec une analyse en termes de déterminisme culturel (Macklin, 1999), comprendre la position des proches implique de saisir la manière dont des réservoirs de signification donnent lieu à différents types de positionnements dans un contexte social et de sens plus large, incluant, entre autres dimensions, le contexte familial, les parcours biographiques des individus impliqués, mais aussi le contexte de soin et les relations aux équipes de soin. De plus, au sein d'une même famille, l'énoncé sur la maladie, sur ce qu'il faut dire ou non au parent âgé, ne fait pas nécessairement consensus.

19. En outre, comme le note Laacher (2014 : 7), les systèmes de solidarité familiale dans les pays d'origine tendent également à s'orienter vers un plus grand « individualisme social », ce que certains observateurs attribuent aux effets de l'urbanisation. t-il de se demander si personnes âgées immigrées et non immigrées ne se trouvent pas confrontées à des enjeux communs du et dans le vieillissement, au-delà des spécificités induites par la situation migratoire. Comme le note Carbonnelle (2009) à propos des recherches menées en Belgique sur les immigrés âgés :

«Il est marquant de constater que cette question est plus fréquemment abordée sous l'angle de perspectives relevant de la sociologie de l'immigration (exil, précarité, altérité, interculturalité) que de la sociologie du vieillissement. À cet égard, s’interroger sur les spécificités des besoins des personnes âgées issues de l'immigration ne devrait pas occulter les similitudes de l'expérience du vieillir et du grand âge entre les cohortes d'origine étrangère et belge... »

Porter un regard croisé sur différentes populations, se distinguant par leurs modes de classement dans l'espace social, offre une vision élargie qui évite une essentialisation des catégories et des personnes. In fine, c'est plus largement la manière d'appréhender la place de l'objet (ou sujet) d'étude au sein du savoir qui est interrogée. Dans le champ des migrations en effet, un débat récurrent se pose : étudie-t-on seulement les populations immigrées pour elles-mêmes, produisant ainsi une connaissance spécifique? Ou le regard porté sur ces populations permet-il, en certaines circonstances du moins, de produire un effet révélateur voire analyseur de processus sociaux plus larges qui, par les effets de la situation migratoire, se trouvent exacerbés?

Ce débat renvoie à un autre, portant sur les catégories utilisées. Un enjeu, et non des moindres, relève des processus de catégorisation et de la manière de nommer la population concernée. Comme le souligne Quiminal (2002 : 9) le chercheur ne peut se passer d'une réflexion sur « le sens et la pertinence des catégories qu'il utilise et leurs effets sociaux ». L'ordre des termes est ainsi à interroger : convientil de parler d'immigrés âgés? De personnes âgées immigrées? Ou cela dépend-il des situations, du viseur émis? La première expression met d'abord l'accent sur la condition d'immigré-e-s, l'âge venant après; la seconde met au premier plan la qualification par l'âge, la condition immigrée venant ensuite.

La complexité de la question est liée aux points de vue nuancés sur ce qui situe, au premier chef, 
les individus dans l'espace social : points de vue où hétéro et auto-définitions ne se superposent pas toujours. Ainsi, l'origine n'est pas nécessairement - même si cela peut l'être - la variable significative mise en avant par les immigrés âgés dans leur rapport aux médecins, comme le montre la manière dont cet ouvrier retraité algérien (73 ans) atteint d'un cancer de la prostate relate son entrevue avec le radiothérapeute. Il explique avoir interrompu le médecin pour lui demander de parler plus lentement " en raison de son grand âge ", mettant ainsi en avant sa condition d'homme âgé plus que celle d'immigré ${ }^{20}$.

L'approche intersectionnelle peut alors, nous semble-t-il, offrir une piste de réflexion intéressante. Cette approche puise son socle théorique dans le Black feminism qui émerge dans les années 1970 aux États-Unis, de la part de militantes noires américaines ne se reconnaissant ni dans le mouvement féministe dominant (composé de femmes blanches de classe moyenne) ni dans le mouvement de libération noir, essentiellement porté par des hommes (Jaunait et Chauvin, 2012). Les théories intersectionnelles permettent de penser l'articulation des rapports sociaux de domination liés notamment aux inégalités de classe, de genre et de «race ». Elles dépassent l'idée d'une simple juxtaposition et addition de désavantages que cumulent des individus en position de dominés du fait de leurs appartenances, pour interroger les effets interactifs de ces formes combinées de domination sur les positions occupées dans l'espace social. Le triptyque le plus couramment retenu, autour des rapports sociaux de classe, de genre et interethniques, laisse souvent de côté les rapports sociaux d'âge et de génération. Pourtant, ces derniers font également partie des grands modes de classement et de hiérarchisation sociaux. En fonction de leur focale, les recherches sur les immigrés âgés gagneraient sans doute à penser cette articulation, ainsi que le propose Ait Ben Lmadani (2007) qui, pour penser la situation des femmes immigrées âgées, articule les théories intersectionnelles avec celles de la reconnaissance sociale.

20. On peut également rappeler ici que plusieurs travaux (Guillou, 2005) ont montré comment le domaine de la santé est globalement perçu par les personnes d'origine étrangère comme confrontant à moins de discriminations que d'autres domaines de la vie sociale (travail, logement, etc.), même si l'existence effective de traitements différentiels est pointée par d'autres recherches (Cognet, Bascougnano, Adam-Véniza, 2009).

\section{Bibliographie}

Ait Ben Lmadani F., 2007. La vieillesse illégitime? Migrantes marocaines en quête de reconnaissance sociale, Thèse de doctorat de sociologie, Université de Paris 7, $371 \mathrm{p}$.

Aвborio A.-M., 1995. Quand le «sale boulot» fait le métier : les aides soignantes dans le monde professionnalisé de l'hôpital, Sciences Sociales et Santé, vol. 13, n³, p. 93-126.

Attias-Donfut C., avec la collaboration de Daveau P., GaLlou R., Rozenkier A., WolfF F.-C., 2006. L'enracinement. Enquête sur le vieillissement des immigrés en France, Paris, Armand Colin, 358 p.

Attias-Donfut C., Gallou R., 2006. L'impact des cultures d'origine sur les pratiques d'entraide familiale, Informations sociales $\mathrm{n}^{\circ} 134(6)$, p. 86-97.

Attias-Donfut C., Delcroix C., 2004. Femmes immigrées face à la retraite, Retraite et société vol., 3, n 43, p. 137-163.

Barou J., Gallou R., 2011 . Vieillir et mourir en Afrique ou en France? Regards croisés de deux générations d'immigrés subsahariens, Gérontologie et société, vol. 139, n 4 , p. 117-145.

Bas-Theron F., Michel M., 2002. Rapport d'activité sur les immigrés vieillissants, Paris, Inspection générale des affaires sanitaires (IGAS), 76 p.

Benveniste A., 2002. Figures politiques de l'identité juive à Sarcelles, Paris, L'Harmattan, 156 p.

Blanc A., 2010. Les Aidants familiaux, Grenoble, Presses universitaires de Grenoble, 255 p.

Boujasson L., 2009. De l'épreuve du travail à l'épreuve de la reconnaissance du cancer en maladie professionnelle, Mouvements, vol.2, $n^{\circ} 58,2009$, p. 56-65.

Carbonnelle S., 2009. Les politiques à l'égard des migrants âgés : la construction d'un nouveau risque social?, Vieillesse et migration, l'Observatoire, [http://www.aifris.org/IMG/pdf/ CARBONNELLE-Sylvie-2.5.pdf].

Cognet M., Bascougnano S., Adam-Veniza E., 2009. Traitement différentiel dans les parcours thérapeutiques. Maladies infectieuses: Vih-Sida, tuberculose, Rapport final, UrmisSoliis, $151 \mathrm{p}$.

Cresson G., 2006. La production familiale de soins et de santé. La prise en compte tardive et inachevée d'une participation essentielle, Recherches familiales, vol. 3, n 1, p. 6-15.

De Sardan J.-P., 1996. L'économie morale de la corruption en Afrique. Politique africaine, vol. 63, p. 97-116.

Dussud F.-X., Le Roho Y., Levy D., Portier J.-F., Rouxel M., 2004. Atlas des immigrés en Bretagne, Insee (direction régionale Rennes), Fasild (direction régionale Bretagne), [en ligne ] : [http://www insee.fr/fr/regions/bretagne/default. asp?page=themes/dossiers/dossier_octant/dossier_atlim/dossier_atlim.htm], consulté le 4 décembre 2012.

Dubus G., Braud F., 2001. Les migrants âgés dans les publications scientifiques francophones, Revue européenne des migrations internationales, vol. 17, n 1, p. 189-197.

FAN R., 1997. Self-determination vs family-determination : two incommensurable principles of autonomy, Bioethics 11 (3-4), p. 310-322. 
Fassin D., Morice A., 2001. Les épreuves de l'irrégularité : les sans-papiers, entre déni d'existence et reconquête d'un statut, in Schnapper D., Exclusions au cour de la cité, Paris, Economia, p 261-309.

Guillou A.-Y., 2005. Expérience migratoire et pratiques thérapeutiques des migrants à Rennes, Rapport d'enquête, ODRIS/RVH35/FASILD. [en ligne] : [http://www.odris.fr/ documents/etudes/Pratiques_Therapeutiques_2005.pdf].

Green N., 2002. Repenser les migrations, Paris, PUF, 138 p.

Gucher C., 2010. Aidants naturels : ambiguïtés et paradoxes d'une notion politiquement construite, in BLANC A. (dir.), Les Aidants familiaux, Grenoble, Presses universitaires de Grenoble, p. 37-49.

Hmed C., 2006. Les résidents vieillissants en foyers pour travailleurs ou les habitants de nulle part, Retraite et société, $n^{\circ} 47$, p. 138-159.

Jaunait A., Chauvin S., 2012. Représenter l'intersection. Les théories de l'intersectionnalité à l'épreuve des sciences sociales, Revue française de science politique, vol. $62, \mathrm{n}^{\circ} 1$, p. 5-20.

Kriegel B., Bougrab J., Salah-Eddine M., 2005. Rapport d'activité sur La condition sociale des travailleurs immigrés âgés, Haut comité à l'intégration (HCI), Paris, 58 p.

LAACHER S., 2014. Femmes immigrées : l'entrée dans la vieillesse, Étude sur le vieillissement des femmes immigrées dans les quartiers en politique de la Ville, pilotée par le Centre de Ressources Politique de la Ville en Essonne, 63 p.

Lardoux J.-M., 2012, La Bretagne est la région française avec le plus faible taux d'immigrés, Info Octant $n^{\circ} 25$, INSEE, [http://www.insee.fr/fr/themes/document.asp?ref_id=19075], consulté le 16 juin 2014.

Macklin R., 1999. Against relativism. Cultural diversity and the search for ethical universals in medicine, Oxford - USA, Oxford University Press, 304 p.

Marche M., 2014. Le contrôle ordinaire et institutionnel de l'expérience du cancer : les limites du «patient-sentinelle », in Pennec S., Le Borgne-Uguen F., Douguet F. (dir.). Les négociations du soin. Les professionnels, les malades et leurs proches, Rennes, PUR, p. 217-224.

Marche M., Pian A., 2014. Vieillir et faire face au cancer. Des ressources objectives aux ressources sélectives, SociologieS, [en ligne] : [http://sociologies.revues.org/4798].
Math A., 2014. L'accès des immigrés âgés aux droits sociaux : éléments pour le débat, in Le vieillissement des populations immigrées, Publication Réso-villes, Centre de ressources politique de la ville Bretagne-Pays-de-la-Loire, 94 p.

Mebtoul M., 2010. La dimension sociopolitique de la production de santé en Algérie, in Cresson G., Mebtoul M. (dir.), Famille et santé, Rennes, EHESP, p. 21-30.

Ménoret M., 1999. Les temps du cancer, Paris, CNRS, 237 p.

MesLin K., 2010. Des logements à part pour les migrants âgés? Réflexion sur la mise à l'écart résidentielle des migrants âgés isolés, Espace, populations, sociétés, vol.1, p. 120-130.

Morillon A., Etiemble A., Veglia P., Folliet D., 2007. Récit historique et mémoire de l'immigration en Bretagne, Tome 1, Rapport et Synthèse de l'étude Histoire et Mémoire de l'Immigration en Bretagne, [en ligne] : [http://www.odris.fr/documents.php], consulté le 2 mars 2011.

Moujoud N., 2003. Partir seules... Heurs et malheurs de marocaines en France, Ghadiva, n 33, p. 93-101.

Pian A., 2014. La fabrique de l'interprétariat auprès des immigrés et étrangers atteints de cancer : la place des proches en question, in Pennec S., Le Borgne-Uguen F., Douget F., Les négociations du soin. Les professionnels, les malades et leurs proches, Rennes, PUR, $283 \mathrm{p}$.

Pian A., 2012. De l'accès aux soins aux trajectoires du mourir. Les étrangers atteints de cancer à l'épreuve des contraintes administratives, Revue européenne des migrations internationales, vol.2, $\mathrm{n}^{\circ} 8$, p. 101-125.

Quiminal C., 2002. Nouvelles mobilités et anciennes catégories, Ville-École-Intégration-Enjeux, n 131, p. 9-20.

RAPport d'ACTIVITÉ DE L'Organisation InTERnATIONALE POUR La Migration (OIM), 2010. L'état de la migration dans le monde en 2010. L'avenir des migrations : renforcer les capacités face aux changements, Genève, OIM, 295 p, [en ligne] : [http://publications.iom.int/bookstore/free/WMR_2010_ FRENCH.pdf], consulté le 20 juin 2014.

SAYAD A., 1999. La double absence. Des illusions de l'émigré aux souffrances de l'immigré, Paris, Seuil, 437 p.

Strauss A., 1992. La trame de la négociation. Sociologie qualitative et interactionniste, Textes réunis et présentés par Isabelle Baszanger, Paris, L'Harmattan, 311 p.

Temine E., 2001. Vieillir en migration, Revue européenne des migrations internationales, vol. $7, \mathrm{n}^{\circ} 1, \mathrm{p} .37-54$. 